\title{
Fire Engine Automated Rescue System
}

\author{
Ajay $\mathrm{R}^{1}$, Monisha $\mathrm{M} \mathrm{V}^{2}$, \\ ${ }^{1}$ Department of Electrical and Electronics Engg Velammal Engineering College Chennai-66, India \\ ${ }^{2}$ Department of Electrical and Electronics Engg Velammal Engineering College Chennai-66, India
}

\begin{abstract}
The heavy traffic congestion has caused much thwarting for any vehicle especially to the fire engines. In order to prevent the enormous amount of property damage and at times loss of life due to the time lag I would like to introduce a new concept called FEARS (Fire engine automated rescue system). The main motive behind this concept is to provide a smooth flow for the fire engines to reach the spot in time and thus minimizing the destruction. The idea behind this scheme is to implement a Intelligent Transportation System which would mechanically control the traffic lights in the path of the fire engine. The fire engine is guided by the main server which furnishes the most suitable route for the fire engine to reach the accident spot. The main server also controls the traffic light according to the fire engine location and thus reaching the spot in time. The server determines the location of the fire accident spot through the sensor systems in the destination which encountered the accident and thus the server walks through the fire engine to the spot. The server calculates the shortest path for the fire engine to the accident spot using Dijkstra algorithm. This scheme is fully automated, thus it finds the fire accident spot, controls the traffic lights, and helps the fire engine to reach spot in time.
\end{abstract}

Keywords: FEARS, Traffic signal control, GPS, GSM, Dijkstra

\section{Introduction}

The loss of property due to the delay in the arrival of fire engines is immense. This delay is mainly caused by the waiting of the fire engine in the traffic signals. It would be of great use to the fire engines if the traffic signals in the path of the accident spot are ON. Thus we propose a new design for automatically controlling the traffic signals and achieving the above mentioned task so that the fire engine would be able to cross all the traffic junctions without waiting. Every traffic junction will have a controller controlling the traffic flow. The traffic junctions are referred to as nodes and each node will have a GSM modem connected to the controller. The nodes are controlled by a main server by sending the control messages to their GSM modems. When a node is controlled and its traffic signal is made to be green for the fire engine to pass through without waiting, it is said to be in ON STATE. For easy access the server maintains a database for each node, and hence each node will have a unique id for addressing it and its GPS co-ordinates are also stored in the database. Thus using these data the fire engine is guided to the fire accident spot by the server through the shortest route.

\section{Fire Engine Automated Rescue System}

Our system consists of four main units, which coordinates with each other and makes sure that fire engine reaches the accident spot without any time lag. Thus our system is divided into following four units,

- The Home Unit

- The Main Server

- The Fire engine Unit

- The Node Circuit.(Traffic Junction Unit).

The home unit installed in each building senses the fire during an accident and sends the location of that building to the main server. The main server finds the nearest fire engine to the accident spot and also the location of the nearest hospital. It calculates the shortest path between the fire engine and accident spot. The server then sends this path to the fire engine. Also using this information, the server controls all the nodes in the path of fire engine and makes it $\mathrm{ON}$, which ensures that the fire engine reaches the spot without delay. The architecture of this system is shown in the fig 1 .

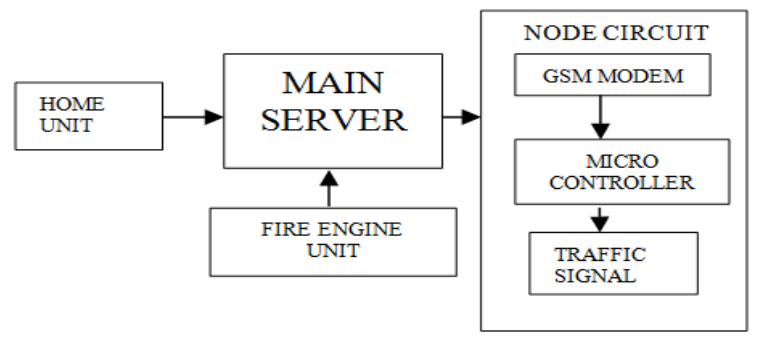

Fig .1 


\section{Home Unit}

According to our system, every building should have a home unit. The home unit consists of a fire sensor, controller, siren, a user interface, GPS system and a GSM module. The fire sensor used in the home unit will continuously sense for any fire in the building [1]. The sensed data is given to the controller.

\section{HOME UNIT}

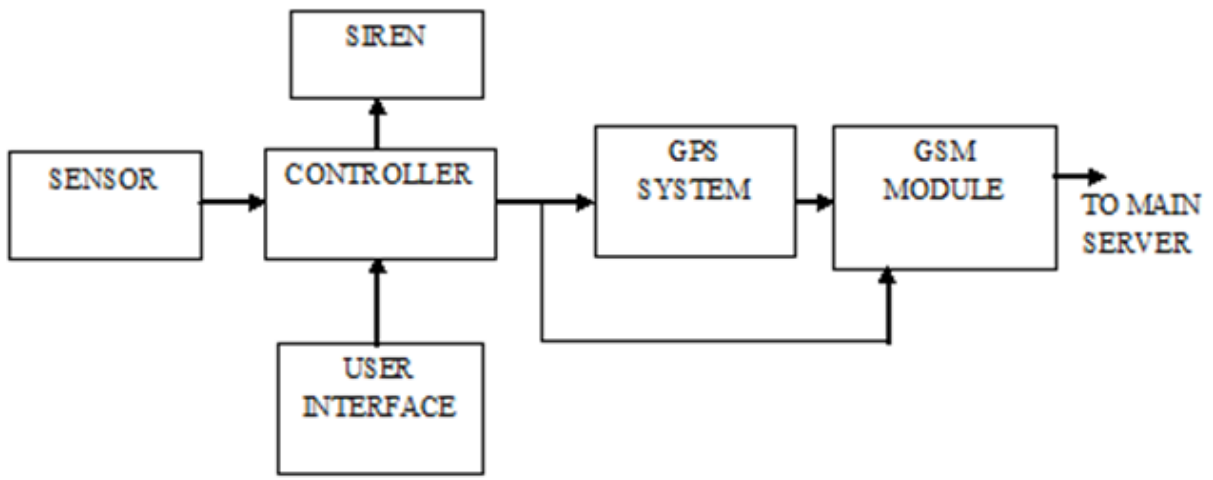

Fig 2

When sensor senses the fire, the controller automatically switches on a siren inside the building. A programmed timer is also triggered. In case of a minor accident, the people inside the building probably would not need the service of the fire engine, and can therefore switch off the siren before the timer counts to zero, by resetting the entire home unit through the user interface, which is connected to the controller. Or else, if the fire is not under control and a fire engine is needed, then the siren is left $\mathrm{ON}$ and when the timer counts to zero, it would trigger both the GSM MODULE and the GPS SYSTEM inside the building unit. The GPS SYSTEM finds out the position of the fire (latitude and the longitude) which is the location of the fire accident spot and gives that data to the GSM MODULE. The GSM MODULE sends this data to the MAIN SERVER whose GSM number is already there in the module as an emergency number. The home unit is shown in the fig 2.

\section{Main Server}

The main server is the central brain and it communicates as well as controls every part of the system [3]. The server objectives can be mainly classified into:

- FINDING THE NEAREST FIRE ENGINE TO THE ACCIDENT SPOT

- SENDING CO-ORDINATES TO THE FIRE ENGINE

- CONTROLLING THE NODES IN THE SHORTEST PATH

\section{A. FINDING THE NEAREST FIRE ENGINE TO THE ACCIDENT SPOT:}

When a fire breaks out in a building, the home unit immediately sends its GPS location to the Main server. The server maintains a database of the fire engines available. The server selects the nearest fire engine to the accident spot using the database containing the details of free and busy fire engines at that point of time. Then the server scans the locations of the free fire engines in the database. It calculates the distance between the accident spot and each fire engine. Then it compares all the distances calculated and selects the nearest fire engine [2].

Therefore for performing the above functions, the server must have the following databases:

- A FIRE ENGINE database - contains list of free and busy fire engines at that time.

- A NODE database - The Main Server allocates a unique ID for each node and has a database to containing all the nodes' IDs, GSM numbers and their GPS co ordinates.

- A Hospital database - containing their locations (GPS coordinates) with their GSM numbers.

\section{1) SHORTEST PATH USING DIJKSTRA:}

As the nodes in the given region are fixed points and the distance between the nodes are predetermined, the shortest path between the nodes can be selected using the DIJKSTRA algorithm. Consider a case when the fire engine travels from Fire station to the accident spot. The database in the server as said earlier contains the node and the distance between the adjacent nodes to which it is connected. The fire station is taken as the source 
and the accident spot is taken as the destination. The node next to the fire station and the node in the path to accident spot must be traced. So that fire station node is taken as source and the accident spot node is taken as destination and the DIJKSTRA algorithm is applied for these nodes. There may be several paths between these nodes and the algorithm finds the shortest path. There may be one way roads along this path, therefore this must be a vector quantity. The server finds nearest node from source and marks it as visited. Then that node is considered as source and the procedure is continued till the destination. Initially, the source doesn't know the distance to destination, so it will be infinite and after complete computation the shortest path along with the distance will be known.

\section{B. SENDING CO-ORDINATES TO THE FIRE ENGINE:}

The server will also find the nearest hospital in addition to calculating the shortest path connecting the fire engine's current location and the accident spot. The shortest path will contain nodes in the path. The server takes the GPS co-ordinates of all the nodes in the shortest path from the NODES database and along with GPS co-ordinates of the accident spot; it transmits it to the fire engine unit in a format specified below.

The nodes' coordinates alone are sent to the fire engine. The format for sending the nodes' coordinates is:

\begin{tabular}{|l|l|l|l|l|l|}
\hline $\mathrm{X} 1, \mathrm{Y}$ & $\mathrm{X} 2, \mathrm{Y}$ & $\cdots$ & $\cdots$ & $\mathrm{Xn}-$ \\
1 & 2 & & & $\mathrm{Xn}, \mathrm{Yn}$ \\
\hline
\end{tabular}

The last coordinates $(\mathrm{Xn}, \mathrm{Yn})$ will indicate the accident location. The coordinates of the accident spot is sent to the nearest hospital.

\section{CONTROLLING THE NODES}

A node can possibly operate in two modes namely, the normal mode and the fire mode. Normal mode is usual traffic control by a micro controller in a junction. In normal mode, traffic flow in each direction of the node will be given equal importance [4]. In the fire mode, the direction in which the fire engine heads is given importance and is kept in the ON state, till the fire engine leaves the junction (node). This is done by

- The node will receive a START SIGNAL from the main server as a control message which contains the direction that must be kept in ON state so that the fire engine can pass through the junction without waiting.

- The direction retrieved from the control message is given to the micro controller.

- That particular direction is kept in the ON state as long as another message (STOP SIGNAL) is received from the main server.

- The STOP SIGNAL is generated when the GPS co-ordinates of the fire engine and the node matches i.e. when the fire engine crosses then node. The node then will return to its normal mode of operation.

1) INTERRUPT SERVICE ROUTINE ALGORITHM

- $\quad$ WAIT FOR THE RECEPTION OF START MESSAGE ALONG WITH THE DATA

- RETRIEVE THE DATA ABOUT THE SIGNAL TO BE MADE GREEN

- MAKE THE CORRESPONDING SIGNAL TO BE GREEN

- $\quad$ WAIT FOR THE RECEPTION OF NEXT MESSAGE OR STOP SIGNAL

- IF THE MESSAGE IS RECEIVED RETURN TO NORMAL MODE

By this way each node in the path to the accident spot is controlled by the server.

This function can be divided into two phases:

2) NODE ACCESS AND CONTROL:

The nodes in the shortest path are accessed and controlled only when the fire engine reaches a distance of around say $1 \mathrm{~km}$ from the node. These locations are stored as the $1 \mathrm{~km}$ markings. Since the signal should not be kept in ON state for a long time, the node access control is done in the following steps:

- The server first plots a map with the nodes needed for the shortest path and makes $1 \mathrm{~km}$ markings for each node.

- The locations of $1 \mathrm{~km}$ markings' (latitude and longitude) are taken from the map and stored in the NODES database.

- When the fire engine's GPS location and location of any one of the $1 \mathrm{~km}$ markings matches, the corresponding GSM ID with the signal direction from the map is taken by the server and is compared with the shortest path nodes' GSM IDs.

- If that node is present in the path, the START SIGNAL is sent to that GSM ID.

- Now, the node is kept in ON state till the fire engine crosses the node. Once it crosses the node, the server sends a STOP SIGNAL to the node which brings the node to normal mode of operation 
- The resolution of the GPS coordinates is that 1 second represents a 101.2ft in latitude and $61.6 \mathrm{ft}$ in longitude. Thus in every comparison with respect to fire engine unit, it is enough to note the GPS coordinate till the accuracy of second's.

Consider a simple case where there is intersection of three junctions as shown in the figure. The two junctions are marked as GS and GN. The distance between these two junctions is less than $1 \mathrm{~km}$. Due to this both the junctions are prone to be considered. Thus for instance if the fire engine is travelling towards the node GS, there would be three $1 \mathrm{~km}$ markings as the path in the direction 1 contains a four way junction. Also as said earlier one of the $1 \mathrm{~km}$ markings of GN comes in the same path to be travelled. When the fire engine approaches the node GS, it also crosses GN's $1 \mathrm{~km}$ marking. Thus this situation is rectified as follows. The node GN's ID is taken by the server from the database, but in actual case this node doesn't need to be controlled. Therefore to eliminate this, the server first compares each node ID taken from the database with the list of nodes' IDs in the shortest path. If the node's ID is present in the list, only then the corresponding node is controlled. So when the GS $1 \mathrm{~km}$ marking is reached, the green light is made ON in the direction 1 and the GN node is not disturbed. Thus, always the every nodes ID is compared with the database containing the nodes ID in shortest path and hence only when the particular ID is matched the node is considered and the traffic light is adjusted according to the need.

\section{CASE NODE SELECTION:}

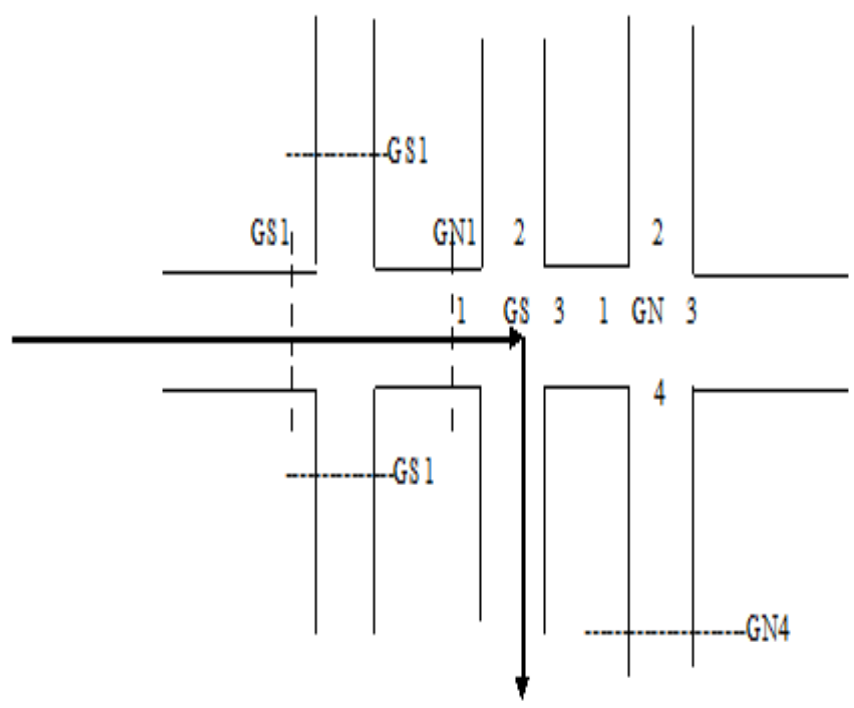

$\longrightarrow$ PATHOF AMBULANCE

GS1-node to be controlled.

GN- node need notbe controlled.

...- $1 \mathrm{~km}$ maxlings of nodes.

The code below is used for implementing the node control. The fire mode is started when an input is got in the C.0 pin of the micro controller from the GSM modem. Consider a signal, similar to message alert in a cell phone, is given to a JK flip flop with its inputs high, so that the output toggles whenever a message is received. Thus when the first message is received, the controller enters into fire mode. The data is collected through port $\mathrm{D}$ and the corresponding direction is put ON. When the next message is received the controller returns back to the normal mode. 
3) CODE FOR CONTROLLING THE NODES:

main () // normal mode

\{

int i, n, $\mathrm{x}$;

$\operatorname{Red}(0)$;

$\operatorname{Red}(1)$;

$\operatorname{Red}(2)$;

$\operatorname{Red}(3)$;

Green $(0)$;

Dela $(60)$;

$\mathrm{i}=0$;

while(1)

\{

$\mathrm{n}=\mathrm{i} \bmod 4$;

Yellow(n);

Dela(5);

$\operatorname{Red}(\mathrm{n})$;

$\mathrm{x}=(\mathrm{n}+1) \bmod 4$;

Green(x);

Dela(60);

i++;

\}

\}

// for switching on yellow signal in direction $\mathrm{x}$ Yellow(int $\mathrm{x}$ )

\{

$\operatorname{switch}(\mathrm{x})$ :

\{

case 1:port A0.A1.A2 $=0$;

port $\mathrm{A} 1=1 ;$ //yellow light in direction 1

break;

case 2: port $\mathrm{A} 5 . \mathrm{A} 6 . \mathrm{A} 7=0$;

port $\mathrm{A} 6=1$;

break;

case 3: port $\mathrm{B} 0 . \mathrm{B} 1 . \mathrm{B} 2=0$;

port $\mathrm{B} 1=1$;

break;

case 4: port B5.B6.B7=0;

port $\mathrm{B} 6=1$;

break;

\}

// for switching on red signal in direction $\mathrm{x}$

$\operatorname{Red}($ int $\mathrm{x}$ )

\{

$\operatorname{switch}(\mathrm{x})$ :

\{

case 1: port $\mathrm{A} 0 . \mathrm{A} 1 . \mathrm{A} 2=0$;

port $\mathrm{A} 2=1 ; / /$ red signal in direction 1

break;

case 2: port A5.A6.A7=0;

port $\mathrm{A} 7=1$;

break;

case 3: port B0.B1.B2=0;

port $\mathrm{B} 2=1$;

break;

case 4: port B5.B6.B7=0; 
port $\mathrm{B} 7=1$;

break;

\}

\}

// for switching on green signal in direction $\mathrm{x}$

Green( int $\mathrm{x}$ )

\{

$\operatorname{switch}(\mathrm{x})$

\{

case 1: port $\mathrm{A} 0 . \mathrm{A} 1 . \mathrm{A} 2=0$;

port $\mathrm{A} 0=1 ;$ //green signal in direction 1

break;

case 2: port A5.A6.A7 $=0$;

port $\mathrm{A} 5=1$;

break;

case 3: port $\mathrm{B} 0 . \mathrm{B} 1 . \mathrm{B} 2=0$;

port $\mathrm{B} 0=1$;

break;

case 4: port B5.B6.B7=0;

port $\mathrm{B} 5=1$;

break;

\}

Dela(int z)

\{

for(int $\mathrm{i}=0 ; \mathrm{i}<\mathrm{z} ; \mathrm{i}++)$

if $($ C. $0==1) \quad / *$ check for reception of START SIGNAL for every second $* /$

Fire();

Call delay; //1 sec

\}

FIre () //fire mode

int $\mathrm{y}=$ port $\mathrm{D} ; / *$ port $\mathrm{D}$ is input port to get the signal direction to be switched $\mathrm{ON}$ for the fire engine*/

$\operatorname{Red}(1) ; \operatorname{Red}(2) ; \operatorname{Red}(3) ; \operatorname{Red}(4) ;$

$\operatorname{switch}(y)$

\{

case 1: Green (1);

break;

case 2: Green (2);

break;

case 3: Green (3);

break;

case 4: Green(4);

break;

\}

/*wait for reception of STOP SIGNAL to return to normal mode*/

while $(\mathrm{C} .0==1)$

\{

\}

\} 


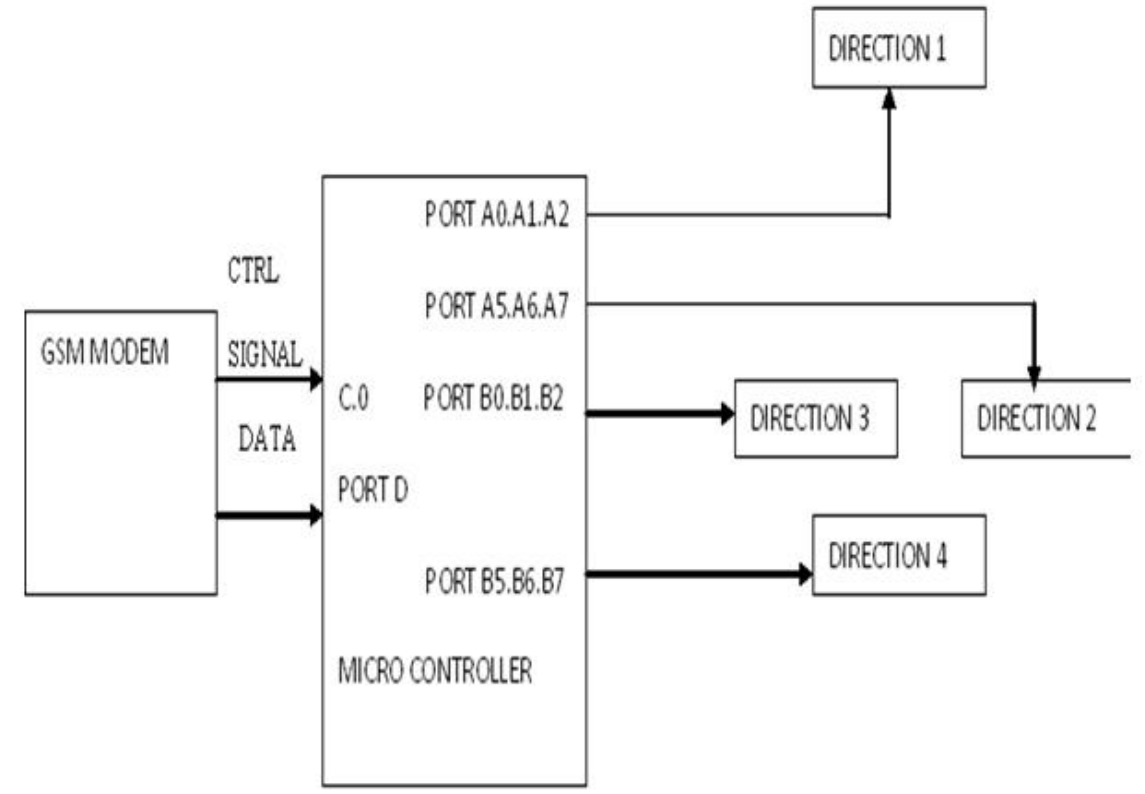

Fire Engine Unit

The fire engine unit has a GPS SYSTEM and a GSM MODEM for transmitting GPS data to the Main Server. The server receives the GPS data sent by the fire engine at regular intervals of time. The server sends the co ordinates of all the nodes' in the path to the fire engine. The last coordinates (Xn, Yn) will indicate the accident location. The fire engine unit on receiving the co-ordinates plots them on to a map with the last co ordinates as the accident spot to get the shortest path to the accident spot.

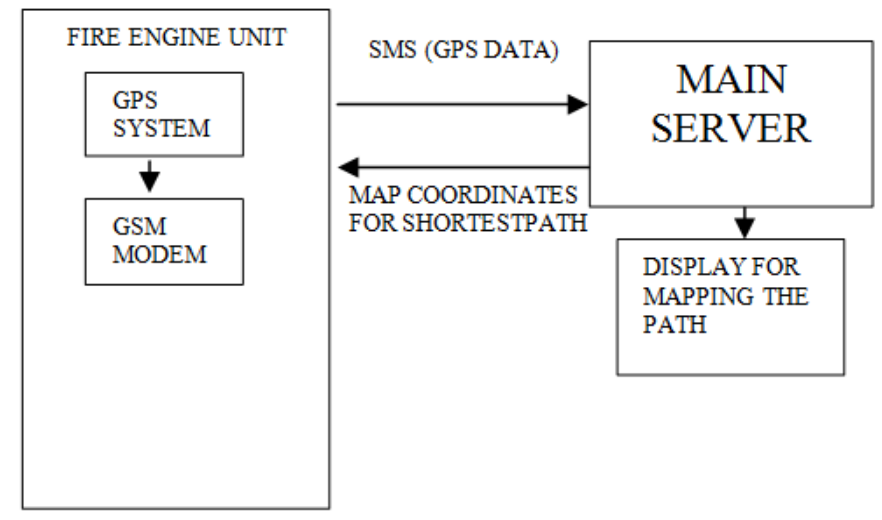

\section{Conclusion}

In this paper, a novel idea is proposed for controlling the traffic signals in favor of fire engines during the accidents. With this system the fire engine can be maneuvered from the fire station to the accident spot without time lag. The FEARS can be proved to be effectual to control fire engines. Thus FEARS if implemented in countries with large population like INDIA can produce better results. The FEARS is more accurate with no loss of time. But there may be a delay caused because of GSM messages since it is a queue based technique, which can be reduced by giving more priority to the messages communicated through the server.

\section{References}

[1]. Wang Wei, Fan Hanbo, Traffic Accident Automatic Detection and Remote Alarm Device

[2]. Zhaosheng Yang. Study on the schemes of traffic signal timing for Priority Vehicles Based on Navigation System, 2000.

[3]. Xiaolin Lu, Develop Web GIS Based Intelligent Transportation Application Systems with Web Service Technology, Proceedings of International Conference on ITS Telecommunications, 2006.

[4]. Katsunori Tawara, Naoto Mukai, Traffic Signal Control by using Traffic Congestion Prediction based on Pheromone Model, Proceedings of 22nd International Conference on Tools with Artificial Intelligence, 2010.

[5]. Mark Allen Weiss, Data structures and Algorithms. 